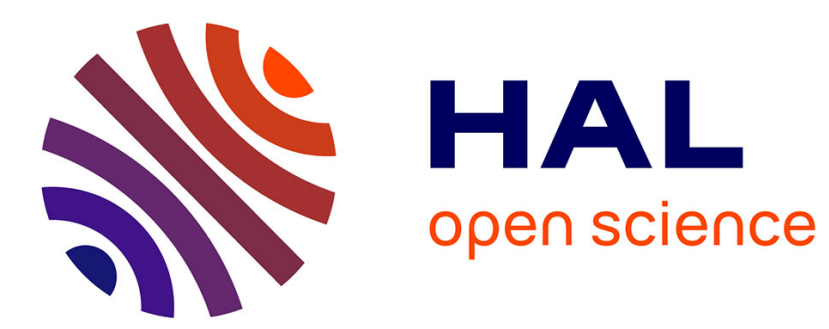

\title{
PHASE CONJUGATION OF THE SOUND WAVE, GENERATED BY THE MOVING SOURCE
}

\author{
V. Strel'Tsov
}

\section{To cite this version:}

V. Strel'Tsov. PHASE CONJUGATION OF THE SOUND WAVE, GENERATED BY THE MOVING SOURCE. Journal de Physique IV Proceedings, 1992, 02 (C1), pp.C1-899-C1-902. 10.1051/jp4:19921196 . jpa-00251161

\section{HAL Id: jpa-00251161 https://hal.science/jpa-00251161}

Submitted on 1 Jan 1992

HAL is a multi-disciplinary open access archive for the deposit and dissemination of scientific research documents, whether they are published or not. The documents may come from teaching and research institutions in France or abroad, or from public or private research centers.
L'archive ouverte pluridisciplinaire HAL, est destinée au dépôt et à la diffusion de documents scientifiques de niveau recherche, publiés ou non, émanant des établissements d'enseignement et de recherche français ou étrangers, des laboratoires publics ou privés. 


\title{
PHASE CONJUGATION OF THE SOUND WAVE, GENERATED BY THE MOVING SOURCE
}

\author{
V.N. STREL'TSOV \\ General Physics Institute of Russian Academy of Science, 38 Vavilov st., 117942 Moscow, USSR
}

\begin{abstract}
We investigate the spatial structure of the contrary sound wave, generated in active parametric layer by the local incident beam, moving along the specimen surface.
\end{abstract}

One of the most interesting directions in modern physical acoustics is the interaction of sound osciliations with some other collective exeitations in solids. Such interaction enables to solve the actual problem of sound phase conjugation (PC) [1-3]. PC can be realized, if phonon-collective interaction $C$ c. phonon-plasmon, phonon-magnon etc.) allows the two-phonon decay of the elemertary excitation with zero quasi-impulse. Indeed, under this condition, if we exelte the collective oscillations with double sound frequency by the external pumping (e.c. electromagnetic field) the travelling incident sound beam in media will induce the generation of the contrary PC wave. Moreover, at high enough pumping level, due to spontaneous two-phonon decay the media becomes unstable. The coupled travelling of incident and contrary waves in unstable regime leads to giant gain in the system. The features of PC process considered essentially depend on system parameters, such as parametric coupling value, dimensions and geometric forms of the active media, beams geometric parameters etc.

In this paper we investigate the spatial distribution of the contrary sound wave, parametrically generated in the layer by the quasi-plane spatially located beam, radiated by the source moving along the specimen surface with steady velocity.

Let the sound source, radiating in self coordinate-system al ong the $z^{\prime}$-ax monochromatic $($ with frequency $\omega)$ quasi-plane wave with some aperture $\mathbf{a}$, be switched on at the moment $t=0$ and move with steady velocity $\nabla$ along the $x$-ax parallel the infinite plane $C$ with normal vector $\vec{n}\left[0,0, n_{z}\right]$, layer of the thickness $l$. In 1 ayer media the effective resonant coupling of the contrary travelling waves exists.

For acoustics field we shall use the hydrodynamics description. For small onough coupling coefficient $\mu$ and not very high velocities $\nabla$. satisfying the condition $\nabla / a * \omega$, the density perturbation $p$ in the sound field in media may be presented as the sum of direct and contrary waves:

$$
\rho=0.5\left[\rho_{1}(x, y, z, t) e^{(\omega t-k z)}+\rho_{2}(x, y, z, t) e^{i(\omega t+k z)}+c .0 .\right]
$$

were $\rho_{1,2}-$ slowly varied along $z, t$ amplitudes $\omega=k c, c$-sound velocity.

Making the transition to the moving coordinate system: $\left(\xi=x-\nabla t, y^{*}=y, z^{*}=2, t^{\prime}=t\right)$ in parabolic approximation for $p$ we derive the following coupled system of reduced equations: 


$$
\begin{aligned}
& \frac{\partial \rho_{2}}{\partial z}-\frac{1}{c}\left(\frac{\partial \rho_{2}}{\partial t}-\nabla \frac{\partial \rho_{2}}{\partial \xi}\right)-\frac{t}{2 k} \cdot \Delta_{1} \rho_{2}=-\frac{1}{2} \cdot i \mu k \rho_{1}^{*} \\
& \frac{\partial \rho_{1}^{*}}{\partial z}+\frac{1}{c}\left[\frac{\partial \rho_{1}^{*}}{\partial t}-\nabla \frac{\partial \rho_{1}^{*}}{\partial \xi}\right]-\frac{i}{2 k} \cdot \Delta_{1} \rho_{1}^{*}=-\frac{1}{2} \cdot \tau \mu k \rho_{2}
\end{aligned}
$$

Neglecting the thermal noise. we have the following initial and boundary conditions :

$$
\rho_{1,2}\left(t\langle 0\rangle=0 ; P_{1,2}\left(\vec{r}_{1}-\infty\right) \rightarrow 0 ; \rho_{1}^{*}(z=0)=\rho_{i n e}^{*}(\xi, y) \theta(t) ;\right.
$$

were $P_{i n e}$ - the incident beam distribution at the entrance surface, $P_{z}(z=l)=0$ - the contrary wave at the back surface 1 s absent.

Further, without restriction of generality, we assume that the input signal $P_{i n e}(\xi, y)$ has the independent distributions along $\xi$ - and $y$-axes and model $\xi$-distribution by the function: $P_{i n c}(\xi, y)=\Phi(y) \exp (-\alpha|\xi|)$ with the characteristic width an $2 / \alpha$. To solve $c 1$ w perform Laplace transform of $P_{1}, \frac{i n}{2}$ time and Fourier transform in space. Then, for images $\tilde{P}_{1,2}$ we get:

with boundary conditions:

$$
\begin{aligned}
& \frac{\partial \tilde{\rho}_{2}}{\partial z}=\left[\frac{p}{c}-i-k_{\xi}-i \frac{k_{1}^{2}}{2 k}\right] \tilde{\rho}_{2}-i \frac{\mu}{2} \tilde{\rho}_{1}^{*} \\
& \frac{\partial \tilde{\rho}_{1}^{*}}{\partial z}=\left[\frac{p}{c}+i \frac{\nabla}{c} k_{\xi}-i \frac{k_{1}^{2}}{2 k}\right] \tilde{\rho}_{1}^{*}-i \frac{\mu-k \tilde{\rho}_{2}}{2}
\end{aligned}
$$

$$
\tilde{\rho}_{1}^{*}(z=0)=\frac{2 \alpha}{p\left(\alpha^{2}+k_{\xi}^{2}\right)} \tilde{\Phi}^{*}\left(k_{y}\right): \tilde{\rho}_{z}(z=0)=0
$$

Solving Ca, for the contrary wave output ampiltude we obtain:

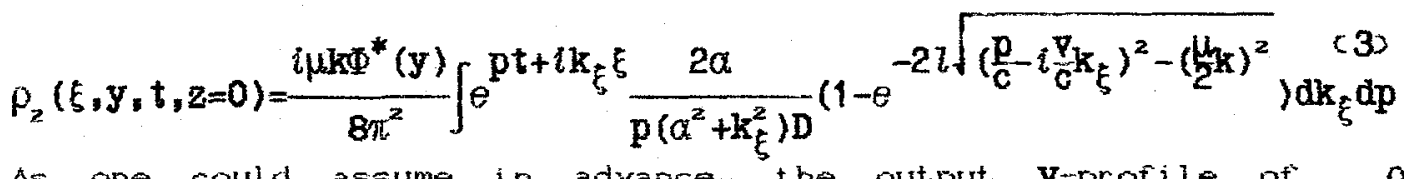
As one could assume in advance, the output $y$-profile of $P_{2}$ reproduces the input $y$ protile $\rho_{1}$.

The integrand in 63 is regular in complex puplares, except the pole $\mathrm{p}=0$ and poles coincidert with roots $\mathrm{D}=0$. For moments $t>2$ l $\mathrm{C}$ the reverse Laplace transform may be carried out with the help of residue theoren : the integration contow is closed in left half of p-plare. Thus, the outpit $s i g n a l$ is presented as a superposition of the modes, corresponding to the above montioned poles. As it was shown in $[4]$ Cses aiso bellow, under the condition $\mu k l<\pi$ all the roots $D=0$ have negative real partis. These modes attenuate ir time and compose the output signal structure in transient regine. The stationary signai, we are inierested in, will be determined by the pole $p=0$. Cubstitution $\mathrm{p}=0$ in 3 immediately yidids:

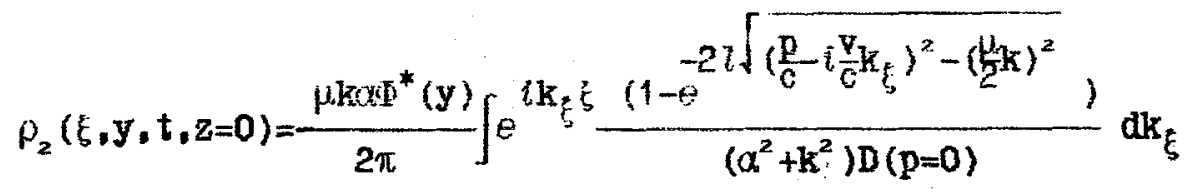


The integrand in (4) as well as in (3) in complex $\mathbf{k}_{\xi}$-plane has the singularities coincident with roots $D(p=0)=0$. For the transverse coordinate interval $|\xi|>2 \nabla l$ C we can again use the path integration $c$ note, that in reality the written interval practically compietely covers the output and input beams contours 3. Now, however, the more detailed treatment of the equation $\mathbf{p}\left(\mathbf{k}_{\xi}\right)=0$ is demanded. This equation was numerically analyzed in [4]. Here we shall treat it in more appropriate for our purpose way.

For the unity of consideration it is convenient to change in (4) the variate $\mathbf{k}_{\xi}$ for $\mathbf{q}=-\mathbf{l} \mathbf{k}_{\xi} \mathbf{v} \mathbf{c}$. Then, making the substitution

$$
0.5 \mu k t=q-\sqrt{q^{2}-\left(\frac{\mu k}{2}\right)^{2}} \quad ; \quad q=0.25 \mu k\left(t+t^{-t}\right)
$$

and separating the real and imaginary parts, we can write the equation $D=0$ as a system of equations for the new complex variate t:

$e^{0.5 \mu k l\left(|t|-|t|^{-1}\right) \cos \varphi}=|t|^{-2} ; 0.5 \mu k l\left(|t|+|t|^{-1}\right) \sin \varphi=-2 \varphi+2 \pi n \quad$ c 5$)$ Note, that (S) is invariant under transiormations : $\left.\left.|| t|\rightarrow| t\right|^{-1} ; \varphi \rightarrow \varphi\right]^{-}$ $[|t| \rightarrow|t| ; \varphi \rightarrow-\varphi ; n \rightarrow-n]$.

From the first equation CS) one can easily see. that the solution with positive real part has module $|t|=1$. At the same time for such |t the second equation will have the solution for $\varphi$ onily under the condition $\mu k l>\pi$. Ihus, is $\mu k l<\pi$, all the roots to ir (5) have negative real parts. In interval $2<\mu k l i \pi$ there exists thee roots with $|t|=1: t_{0}=-1$ and two complex conjugated roots, circling $c$ when $\mu k l \rightarrow \pi$ in the left half of $q$-plane from $t_{0}=-1$ to $t$ and $-i$ correspondingly. For the length $l$ far enough from the criticai ore Mkl<2 the root with $|t|=1$ is single: $t_{0}=-1$. For such small iength there exists also two real negative roots, which approach 6 when $\mu k l \rightarrow 2$ 3 to $t_{0}=-1$. Another roots are complex and as can be shown have $|t| \geqslant 10<|t| \leqslant 0.13$.

Retuning to (4), we take into account, that for variant $q$ the integration contours rotate by the angle $\pi$ a about $k_{\text {- }}$ contours. Ther, for $\xi>2 v l \wedge c$ the integration path will be closed in right half of q-plane. For the lengths $\mu k l<\pi$, as it follows from the above analyses, the roots $D(q)=0$ lie in lefi half ard the single pole making a contribution in integral is $q=\alpha$. Finally, ior $\zeta>0$ we obtair

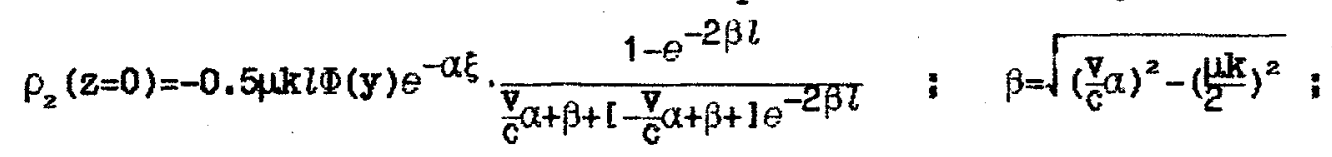

We see, that the contrary beam, moving together with the ineident beam, has the front profile, coincident with the irort profile of the last ore. We shall look through two limits:

1. "Narrow" beam: Po.cukk?2.

$$
\rho_{2}(\mathrm{z}=0)=-\alpha^{*}(\mathrm{y})\left(1-e^{-2 \nabla \alpha l<c}\right) e^{-\alpha} \cdot \mu k c \cdot 4 \nabla \alpha
$$

The output ampiitude is decreased in compare with input incident beam amplitude.

2. "Wi de" beam: pa/cqule?

$$
\rho_{2}(z=0)=-\Phi^{*}(y) e^{-\alpha \xi} \sin (\mu k l-2)-[\cos (\mu k l-2)+\sin (\mu k l-2) \cdot 2 v \alpha \mu k c]
$$

Far from the edge of the stability Hkl《1 the signal is week. The 
growth of the effective layer thickness plkl increases the output

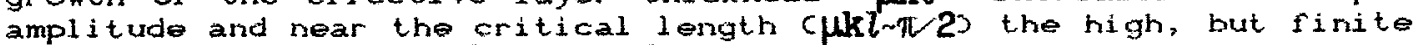
gain takes place $p_{2}(z=0) / p_{\text {inc }}$ flck $2 \nabla \alpha$.

Now we shall come to the back front consideration: $\xi<-2 l$. It's convenient to introduce the new variable $\eta=\xi+2 v l, c \leq 0$. Closing the integration contour in the left half of q-plane, we obtain cnow all the roots to of $D$ make contribution to integrals:

$$
\begin{gathered}
\rho_{2}(z=0)=-0.5 \mu k^{*}(\mathrm{y})\left\{\frac{\left(1-e^{-2 \beta l}\right) e^{-2 a \nabla l-c}}{\frac{\nabla}{c} \alpha+\beta+\left(\frac{\nabla}{c} \alpha+\beta\right) e^{-2 \beta l}} \cdot e^{\alpha \eta}-\right. \\
\left.-\sum \frac{\left(\alpha \frac{c}{\nabla}\right)\left(t_{0}-t_{0}^{-1}\right)^{2} e^{-0.5 \mu k l\left(t_{0}+t_{0}^{-2}\right)}}{\left[\alpha^{2}-\left(0.25 \mu k_{\bar{v}}^{c}\right)^{2}\left(t_{0}+t_{0}^{-1}\right)^{2}\right]\left[2+0.5 \mu k l\left(t_{0}+t_{0}^{-1}\right)\right]} \cdot e^{\frac{\mu k c}{4 \nabla} \eta\left(t_{0}+t_{0}^{-1} \eta\right.}\right\}
\end{gathered}
$$

one can easily veriry, using tho above analysis, that the complex roots with $|t| \neq 1$ al ways make the negligible contributions to (6). Up to the Iength $\mu k l-2$ the real negative roots also can be neglected. Thereby, under the condition 2avres1 the first term in 60 will be dominant and all the results obtained for front profile are preserved. Thus, for the lengths, far from criticai and for not very small transverse dimensions of the incident beam the back front of the contrary wave reproduces the back front of the incident beam, and we may speak about conjugation.

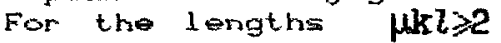
arises. We shall look agair through two limits. 1. "Narrow" beam.

$$
\rho_{2}(z=0)=-\frac{\mu k c}{4 \nabla \alpha} \cdot \Phi^{*}(y)\left\{\left(1-e^{-2 v a l-c}\right) e^{a \eta}+16 \sin ^{2} \varphi \frac{e^{\ln \cos \varphi(l-\eta \sigma \alpha v)}}{2+\mu k l \cos \varphi}\right\}
$$

The second term moriotonously grows, when the length $l$ increases from

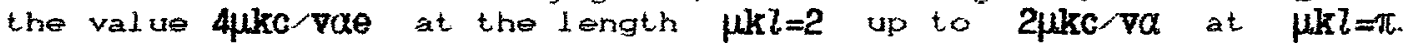
Simultaneously its width also rises and near the edge of stability becomes irfirite. Thus, beyond the movirig contrary beam the sound

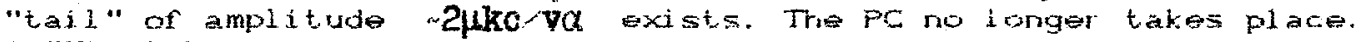
e. "Wi ds" beam.

$$
\begin{aligned}
\rho_{z}(z=0)= & -\Phi^{*}(y)\left\{\frac{\sin (\mu k l-2) e^{-2 v l \alpha-c}}{\cos (\mu k l-2)-(2 v \alpha \mu k c) \sin (\mu k l-2)} \cdot e^{\alpha \eta}\right. \\
& \left.-4 \cdot \frac{\nabla \alpha e^{l} \mu \cos p(l-\eta \omega-v)_{\sin }^{2} \varphi}{c\left[\alpha^{2}-\left(0.5 \mu k \frac{c}{p} \cos \varphi\right)^{2}\right](2+\mu k l \cos \varphi)}\right\}
\end{aligned}
$$

As in previous case, the second term is small at the iength Hkl Its amplitude and width grow as length increases and near the edge of stability beyond the moving contrary beam the track of the amplitude -2 kkc $2 \alpha$ is formed. Note, that in contrast with narrow beam now the gain takes place: $p_{2}(2=0) / p_{i n c}>1$.

\section{REFERENCES}

1\%. Strel'tsov V. N. Kvantovaya Elestron. (Moscow) $13,2144,(1986)$.

2\% Bryser A.P. Strel*tsov V.M. Proc. of 1 st France Acoust. Congr. Coll. de Phys., suppl. fit $2,51,6 z-85,(1990)$.

3.. Erysev A.P. et al. Optical sacoustical Review.1.107(1900).

4.\% Bobr off D.L., Haus H. A., J. Appl. Fhys. 38, 390, (1967). 